\title{
A Uranium Tri-Rhenium Triple Inverse Sandwich Compound
}

Michael A. Boreen, ${ }^{\dagger}$, Trevor D. Lohrey, ${ }^{\dagger}{ }^{\ddagger}$ Guodong Rao, ${ }^{\S}$ R. David Britt, ${ }^{\S}$ Laurent Maron, ${ }^{\#}$ and John Arnold ${ }^{\dagger, \ddagger}, *$

'Department of Chemistry, University of California, Berkeley, California 94720, USA

${ }^{+}$Chemical Sciences Division, Lawrence Berkeley National Laboratory, Berkeley, California 94720, USA

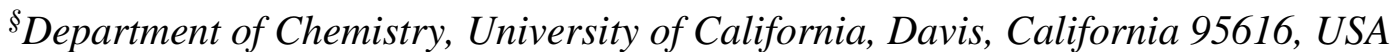

${ }^{\#}$ LPCNO, Université de Toulouse, INSA Toulouse, 135 Avenue de Rangueil, 31077 Toulouse, France

*Email: arnold@berkeley.edu

\section{Supporting Information}

Experimental procedures

S2-S3

UV-Vis spectra

NMR spectra

X-ray crystallographic details

S8-S12

EPR spectroscopy

Computational details and coordinates of optimized structures

S14-S24

References

S25-S27 


\section{Experimental procedures}

General Considerations: Unless otherwise noted, all reactions were performed using standard Schlenk line techniques under an atmosphere of nitrogen or argon or in an MBraun inert atmosphere glove box under an atmosphere of nitrogen. Glassware and Celite ${ }^{\circledR}$ were stored in an oven at $\mathrm{ca} .150{ }^{\circ} \mathrm{C}$ for at least $3 \mathrm{~h}$ prior to use. Molecular sieves ( $4 \AA$ ) were activated by heating to $200{ }^{\circ} \mathrm{C}$ overnight under vacuum prior to storage in a glove box. NMR spectra were recorded on Bruker AV-300 and AV-500 spectrometers. ${ }^{1} \mathrm{H}$ chemical shifts are given relative to residual solvent peaks and are recorded in units of parts per million (ppm). FT-IR samples were prepared as Nujol mulls pressed between $\mathrm{KBr}$ plates, with data collected with a Nicolet iS10 FT-IR spectrometer. Melting points were determined using sealed capillaries prepared under nitrogen on an OptiMelt automated melting point system. Elemental analyses were determined at the Microanalytical Facility at the College of Chemistry, University of California, Berkeley. UV-Vis measurements were performed on a Varian Cary® $50 \mathrm{UV}-$ Vis Spectrophotometer. A two mm path length quartz cell was used, and a blank was subtracted from each run.

Materials: Diethyl ether, $n$-hexane, and THF were purified by passage through columns of activated alumina and degassed by sparging with nitrogen. $\mathrm{C}_{6} \mathrm{D}_{6}$ was vacuum-transferred from a flask containing sodium/benzophenone, degassed with three freeze-pump-thaw cycles, and stored over molecular sieves. $\mathrm{UI}_{3}(1,4 \text {-dioxane })_{1.5},{ }^{1} \mathrm{UI}_{3},{ }^{2} \mathrm{UCl}_{4},{ }^{3} \mathrm{KC}_{8},{ }^{4}$ and $\left[{ }^{n} \mathrm{Bu}_{4} \mathrm{~N}\right]\left[\mathrm{B}\left(\mathrm{C}_{6} \mathrm{~F}_{5}\right)_{4}\right]^{5}$ were synthesized according to literature procedures. $\mathrm{Na}\left[\mathrm{Re}\left(\eta^{5}-\mathrm{Cp}\right)(\mathrm{BDI})\right]^{6}$ was made according to literature procedures and recrystallized from a mixture of diethyl ether and toluene at room temperature prior to use. All other chemicals were purchased from commercial sources and used as received.

(THF)U[( $\boldsymbol{\mu}-\boldsymbol{\eta}^{\mathbf{5}}: \boldsymbol{\eta}^{\mathbf{5}}$-Cp)Re(BDI)]3 (1·THF): $\mathrm{UI}_{3}(1,4$-dioxane) 1.5 (55 mg, $0.073 \mathrm{mmol}, 1.0$ equiv) and $\mathrm{Na}\left[\mathrm{Re}\left(\eta^{5}-\mathrm{Cp}\right)(\mathrm{BDI})\right]$ (152 $\mathrm{mg}, 0.219 \mathrm{mmol}, 3.0$ equiv) were added as solids to a $20 \mathrm{~mL}$ scintillation vial. Diethyl ether $(3 \mathrm{~mL})$ was added, and the reaction mixture was stirred at room temperature for $1 \mathrm{~h}$. THF (15 drops) was added, and the reaction mixture was stirred for an additional 15 minutes at room temperature. Volatiles were removed in vacuo, and the product was dissolved in $16 \mathrm{~mL}$ of hexane, filtered through Celite, concentrated, and cooled to $-40{ }^{\circ} \mathrm{C}$, resulting in the formation of dark red crystals. Concentration and cooling of the supernatant yielded a second crop of crystals in a similar fashion (140 mg combined, $0.060 \mathrm{mmol}, 83 \%$ yield). X-ray quality crystals of 1-THF were grown from hexane at room temperature. Mp $142-146{ }^{\circ} \mathrm{C}$ (decomp.); ${ }^{1} \mathrm{H}$ NMR (500 MHz, C6 $\left.\mathrm{D}_{6}\right): \delta 7.99\left(\mathrm{~s}, 3 \mathrm{H}, \mathrm{HC}\left[\mathrm{C}\left(\mathrm{CH}_{3}\right)(\mathrm{NDipp})\right]_{2}\right), 6.95\left(\right.$ broad, $\left.12 \mathrm{H}, \mathrm{Dipp} \mathrm{HC}\left(\mathrm{CH}_{3}\right)_{2}\right)$, 5.51 (d, J = 7.6 Hz, 12H, Dipp aryl), 3.93 (t, J = 7.5 Hz, 6H, Dipp aryl), 2.09 (d, J = 6.8 Hz, $36 \mathrm{H}$, Dipp $\left.\mathrm{HC}\left(\mathrm{CH}_{3}\right)\left(\mathrm{CH}_{3}\right)\right), 1.97\left(\mathrm{~d}, \mathrm{~J}=6.4 \mathrm{~Hz}, 36 \mathrm{H}, \operatorname{Dipp} \mathrm{HC}\left(\mathrm{CH}_{3}\right)\left(\mathrm{CH}_{3}\right)\right), 0.76(\mathrm{~s}, 18 \mathrm{H}$, $\left.\mathrm{HC}\left[\mathrm{C}\left(\mathrm{CH}_{3}\right)(\mathrm{NDipp})\right]_{2}\right),-10.89$ (broad, 4H, THF), -24.24 (broad, 4H, THF), -31.76 (s, 15H, $\mathrm{C}_{5} H_{5}$ ); $\mu_{e f f}=3.1 \mu_{B}\left(293 \mathrm{~K}\right.$, Evans method, $\mathrm{C}_{6} \mathrm{D}_{6}$; note: the accuracy of this measurement is limited due to presence of small quantities of a paramagnetic impurity, $\operatorname{Re}\left(\eta^{5}-\mathrm{Cp}\right)(\mathrm{BDI})$, which was factored into 
the calculation of $\mu_{\text {eff }}$ based on an estimate of its relative concentration); IR: $3053(\mathrm{w}), 1583(\mathrm{w})$, 1539 (s), 1435 (s), 1358 (m), 1323 (s), 1304 (m), 1252 (m), 1223 (w), 1176 (m), 1157 (w), 1102 (m), 1055 (w), $1041(\mathrm{~m}), 1018(\mathrm{~m}), 932$ (w), $870(\mathrm{w}), 844(\mathrm{w}), 792(\mathrm{~m}), 783(\mathrm{~m}), 759(\mathrm{~s}), 717$ (m).Anal. Calcd (\%) for $\mathrm{C}_{106} \mathrm{H}_{146} \mathrm{~N}_{6} \mathrm{ORe}_{3} \mathrm{U}(\mathbf{1} \cdot \mathbf{T H F})$ : C, 54.95; H, 6.35; N, 3.63. Found: C, 55.02; H, 6.49; N, 3.52 .

(1,4-dioxane)U[( $\boldsymbol{\mu}-\boldsymbol{\eta}^{\mathbf{5}}: \boldsymbol{\eta}^{\mathbf{5}}$-Cp) $\left.\operatorname{Re}(\mathrm{BDI})\right]_{3}$ (1·diox): $\mathrm{UI}_{3}(1,4 \text {-dioxane) })_{1.5}$ (18 $\mathrm{mg}, 0.024 \mathrm{mmol}, 1.0$ equiv) and $\mathrm{Na}\left[\operatorname{Re}\left(\eta^{5}-\mathrm{Cp}\right)(\mathrm{BDI})\right](50 \mathrm{mg}, 0.072 \mathrm{mmol}, 3.0$ equiv) were added as solids to a $20 \mathrm{~mL}$ scintillation vial. Diethyl ether $(2 \mathrm{~mL})$ was added, and the dark red reaction mixture was stirred at room temperature for $1 \mathrm{~h}$. Volatiles were removed in vacuo, and the product was dissolved in 12 $\mathrm{mL}$ of hexane, filtered through Celite, concentrated, and cooled to $-40{ }^{\circ} \mathrm{C}$, resulting in the formation of dark red crystals $(35 \mathrm{mg}, 0.015 \mathrm{mmol}, 62 \%$ yield). X-ray quality crystals of $\mathbf{1} \cdot \mathbf{d i o x}$ were grown from a solution of ether/hexane (1:1.5 ratio), after decreasing the volume briefly under reduced pressure, at $-40{ }^{\circ} \mathrm{C} .{ }^{1} \mathrm{H}$ NMR $\left(300 \mathrm{MHz}, \mathrm{C}_{6} \mathrm{D}_{6}\right): \delta 8.15\left(\mathrm{~s}, 3 \mathrm{H}, H \mathrm{C}\left[\mathrm{C}\left(\mathrm{CH}_{3}\right)(\mathrm{NDipp})\right]_{2}\right)$, 7.20 (overlaps residual $\mathrm{C}_{6} \mathrm{D}_{5} \mathrm{H}$ peak, $12 \mathrm{H}$, Dipp $\left.H \mathrm{C}\left(\mathrm{CH}_{3}\right)_{2}\right), 5.33(\mathrm{~d}, \mathrm{~J}=7.6 \mathrm{~Hz}, 12 \mathrm{H}$, Dipp aryl), 3.65 (t, J = 7.6 Hz, 6H, Dipp aryl), $2.13\left(\mathrm{~d}, \mathrm{~J}=6.8 \mathrm{~Hz}, 36 \mathrm{H}\right.$, Dipp $\mathrm{HC}\left(\mathrm{CH}_{3}\right)\left(\mathrm{CH}_{3}\right)$ ), 2.09 (d, J = $\left.6.5 \mathrm{~Hz}, 36 \mathrm{H}, \mathrm{Dipp} \mathrm{HC}\left(\mathrm{CH}_{3}\right)\left(\mathrm{CH}_{3}\right)\right), 0.88$ (s, 18H, HC[C(CH 3$\left.\left.)(\mathrm{NDipp})\right]_{2}\right),-12.61$ (broad, dioxane), $-31.73\left(\mathrm{~s}, 15 \mathrm{H}, \mathrm{C}_{5} \mathrm{H}_{5}\right)$. Further characterization was not performed due to the lability, and therefore variable dissociation, of the 1,4-dioxane ligand upon storage of solid samples of $\mathbf{1} \cdot \mathbf{d i o x}$ at room temperature.

(DMAP)U[( $\left.\left.\boldsymbol{\mu}-\boldsymbol{\eta}^{\mathbf{5}}: \boldsymbol{\eta}^{\mathbf{5}}-\mathbf{C p}\right) \operatorname{Re}(\mathbf{B D I})\right]_{3}$ (1·DMAP): $\mathrm{UI}_{3}(1,4 \text {-dioxane })_{1.5}$ (17 mg, $0.023 \mathrm{mmol}, 1.0$ equiv) and $\mathrm{Na}\left[\operatorname{Re}\left(\eta^{5}-\mathrm{Cp}\right)(\mathrm{BDI})\right](47 \mathrm{mg}, 0.068 \mathrm{mmol}, 3.0$ equiv) were added as solids to a $20 \mathrm{~mL}$ scintillation vial. Diethyl ether $(2 \mathrm{~mL})$ was added, and the reaction mixture was stirred at room temperature for $1 \mathrm{~h}$. 4-(Dimethylamino)pyridine $(3.0 \mathrm{mg}, 0.025 \mathrm{mmol}, 1.1$ equiv) was added as a solid, and the reaction mixture was stirred for an additional 45 minutes at room temperature. Volatiles were removed in vacuo, and the product was dissolved in $26 \mathrm{~mL}$ of hexane, filtered through Celite, concentrated, and cooled to $-40{ }^{\circ} \mathrm{C}$, resulting in the formation of dark red crystals (38 mg, $0.016 \mathrm{mmol}, 71 \%$ yield). X-ray quality crystals of 1-DMAP were grown from hexane at room temperature. Mp 164-168 ${ }^{\circ} \mathrm{C}$ (decomp.); ${ }^{1} \mathrm{H}$ NMR $\left(500 \mathrm{MHz}, \mathrm{C}_{6} \mathrm{D}_{6}\right): \delta 7.75$ (s, 3H, $\left.H C\left[C\left(\mathrm{CH}_{3}\right)(\mathrm{NDipp})\right]_{2}\right), 6.55\left(\mathrm{~m}, 12 \mathrm{H}, \operatorname{Dipp~} H \mathrm{C}\left(\mathrm{CH}_{3}\right)_{2}\right), 6.00(\mathrm{~d}, \mathrm{~J}=7.4 \mathrm{~Hz}, 12 \mathrm{~Hz}$, Dipp aryl), $4.72\left(\mathrm{t}, \mathrm{J}=7.6 \mathrm{~Hz}, 6 \mathrm{H}\right.$, Dipp aryl), $2.03\left(\mathrm{~d}, \mathrm{~J}=6.7 \mathrm{~Hz}, 36 \mathrm{H}\right.$, Dipp $\left.\mathrm{HC}\left(\mathrm{CH}_{3}\right)\left(\mathrm{CH}_{3}\right)\right), 1.64(\mathrm{~d}, \mathrm{~J}=$ $\left.6.3 \mathrm{~Hz}, 36 \mathrm{H}, \operatorname{Dipp~} \mathrm{HC}\left(\mathrm{CH}_{3}\right)\left(\mathrm{CH}_{3}\right)\right), 0.63$ (s, 18H, $\left.\mathrm{HC}\left[\mathrm{C}\left(\mathrm{CH}_{3}\right)(\mathrm{NDipp})\right]_{2}\right),-2.49$ (broad, 2H, DMAP aryl), -3.25 (s, 6H, DMAP N(CH ( $\left._{2}\right)$ ), -12.81 (broad, 2H, DMAP aryl), -31.58 (s, 15H, $\left.\mathrm{C}_{5} H_{5}\right) \mu_{e f f}=3.2 \mu_{B}\left(293 \mathrm{~K}\right.$, Evans method, $\mathrm{C}_{6} \mathrm{D}_{6}$; note: the accuracy of this measurement is limited due to presence of small quantities of a paramagnetic impurity, $\operatorname{Re}\left(\eta^{5}-\mathrm{Cp}\right)(\mathrm{BDI})$, which was factored into the calculation of $\mu_{\text {eff }}$ based on an estimate of its relative concentration); IR: 3048 (w) 1611 (s), 1584 (w), 1538 (s), 1435 (s), 1358 (s), 1322 (s), 1305 (m), 1250 (s), 1228 (m), 1176 (m), $1156(\mathrm{w}), 1101(\mathrm{w}), 1055(\mathrm{w}), 1040(\mathrm{~m}), 1019(\mathrm{~m}), 997(\mathrm{~s}), 949(\mathrm{w}), 933(\mathrm{w}), 846(\mathrm{w}), 807(\mathrm{w})$, $785(\mathrm{~m}), 758(\mathrm{~s}), 717(\mathrm{~m})$. 


\section{UV-Vis spectra}

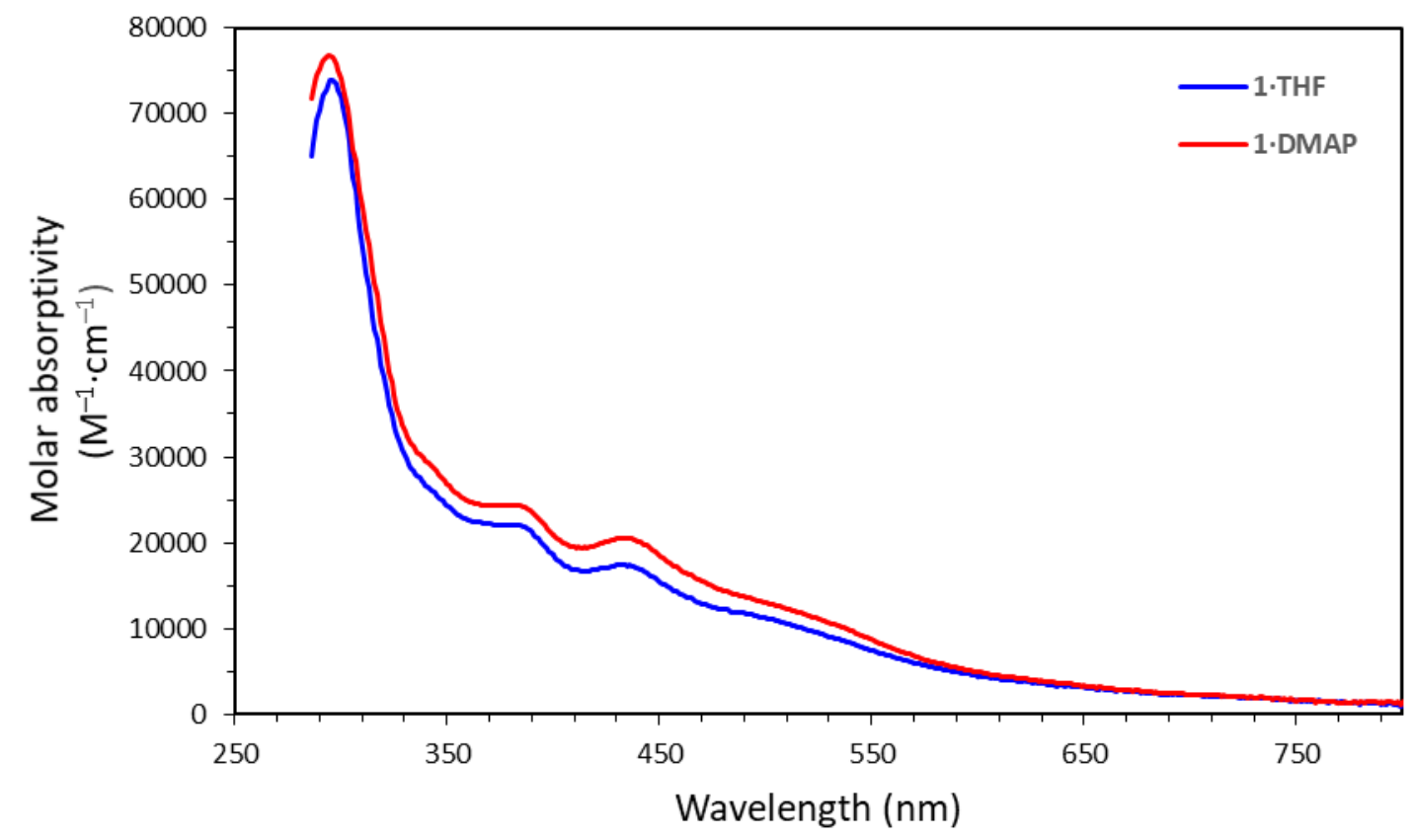

Figure S1. UV-Vis spectra of 1·THF (blue) and 1·DMAP (red) in toluene. 


\section{NMR spectra}

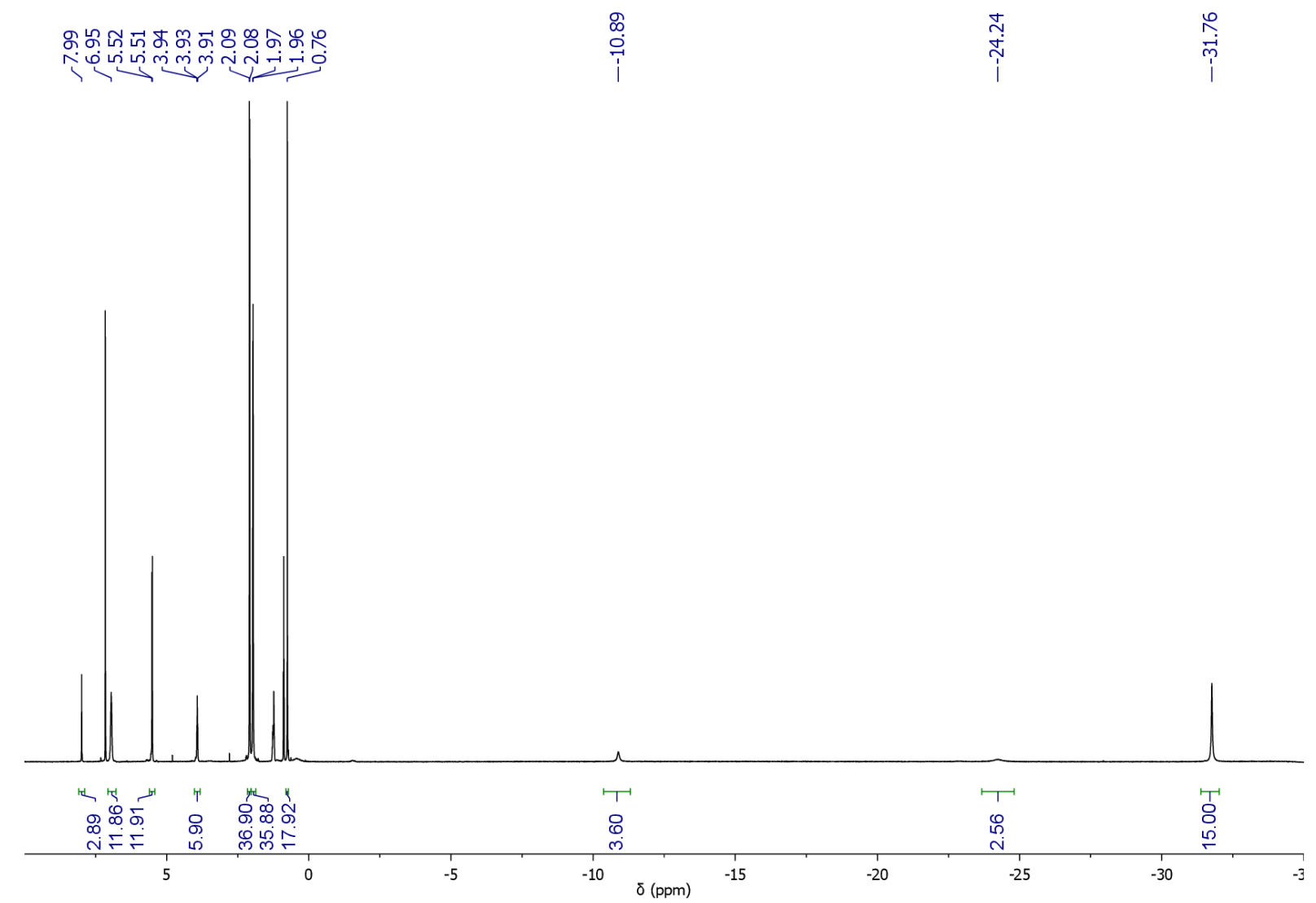

Figure S2. ${ }^{1} \mathrm{H}$ NMR spectrum of $\mathbf{1} \cdot \mathbf{T H F}$ in $\mathrm{C}_{6} \mathrm{D}_{6}$. 


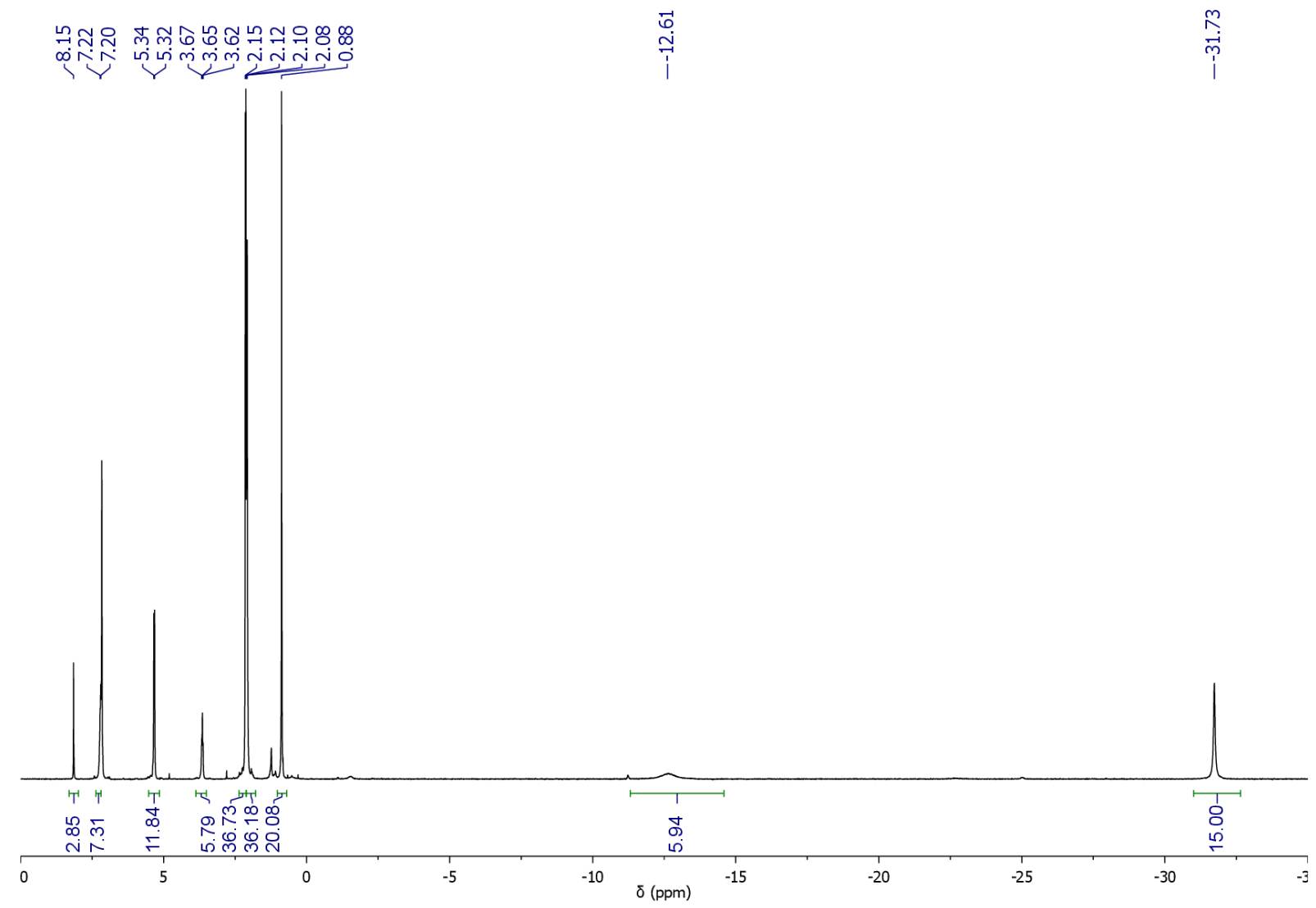

Figure S3. ${ }^{1} \mathrm{H}$ NMR spectrum of $\mathbf{1} \cdot$ diox in $\mathrm{C}_{6} \mathrm{D}_{6}$. 


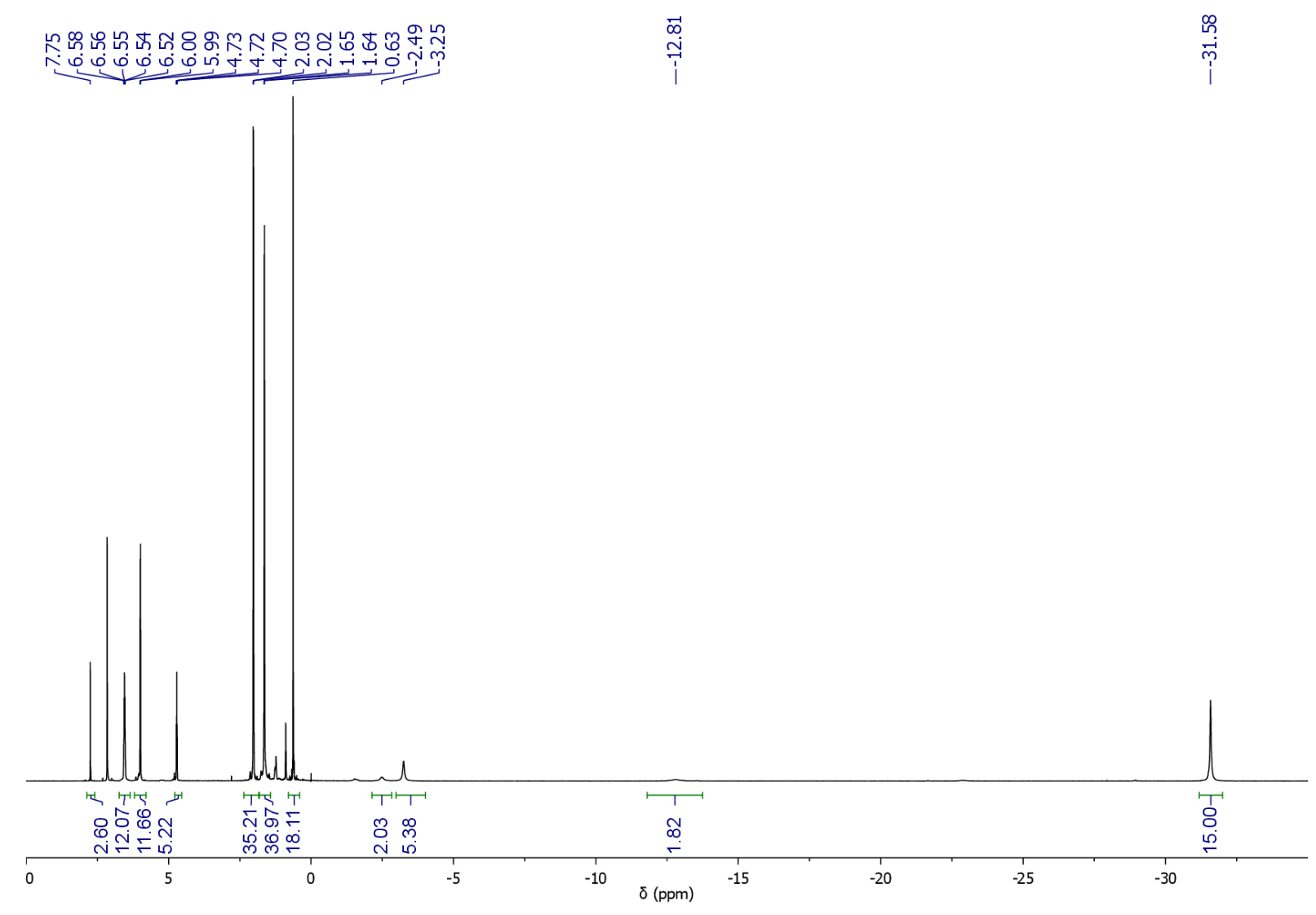

Figure S4. ${ }^{1} \mathrm{H}$ NMR spectrum of 1.DMAP in $\mathrm{C}_{6} \mathrm{D}_{6}$. 


\section{X-ray crystallographic details}

In a dry nitrogen glovebox, samples of single crystals of $\mathbf{1} \cdot \mathbf{d i o x}, \mathbf{1} \cdot \mathbf{T H F}$, and 1·DMAP were coated in Paratone-N oil prior to transport to diffraction facilities, where they were evaluated by polarized light microscopy and mounted on a Kaptan loop (for 1·diox and 1·THF) or on a MiTeGen $10 \mu \mathrm{m}$ aperture Dual-Thickness MicroMount (for 1·DMAP). X-ray diffraction data for $\mathbf{1 \cdot d i o x}$ and 1-THF were collected at CheXray, Berkeley, CA, using a Bruker APEX II QUAZAR instrument outfitted with a monochromated radiation source (for 1·THF) or on a Rigaku XtaLAB P200 equipped with a MicroMax-007 HF microfocus rotating anode and a Pilatus 200K hybrid pixel array detector (for $\mathbf{1} \cdot \mathbf{d i o x}$ ). Data for $\mathbf{1} \cdot \mathbf{d i o x}$ and $\mathbf{1} \cdot \mathbf{T H F}$ were collected using Mo K $\alpha$ radiation $(\lambda$ $=0.71073 \AA$ ). X-ray diffraction data for 1-DMAP were collected at the Advanced Light Source (ALS), Lawrence Berkeley National Lab, Berkeley, CA, station 12.2.1 using a silicon monochromated beam of $17 \mathrm{keV}(\lambda=0.7288 \AA)$ synchrotron radiation. All data collections were conducted at $100 \mathrm{~K}$, with the crystals cooled by a stream of dry nitrogen. For 1·THF and 1·DMAP, Bruker APEX2 or APEX3 software was used for the data collections, Bruker SAINT software was used to conduct the cell refinement and data reduction procedures, ${ }^{7}$ and absorption corrections were carried out by a multi-scan method utilizing the SADABS program. ${ }^{8}$ For $\mathbf{1} \cdot \mathbf{d i o x}$, Crys Alis ${ }^{\text {Pro }}$ was used for the data collections and data processing, including a multi-scan absorption correction applied using the SCALE3 ABSPACK scaling algorithm within CrysAlis ${ }^{\text {Pro }}{ }^{9}$ Initial structure solutions were found using direct methods (SHELXT), ${ }^{10}$ and refinements were carried out using SHELXL-2014. ${ }^{11-13}$ Thermal parameters for all non-hydrogen atoms were refined anisotropically. Thermal ellipsoid plots were made using Mercury. ${ }^{14}$ All structures have been deposited to the Cambridge Crystallographic Data Centre (CCDC), with deposition numbers 1893400 (1·THF), $1893401(\mathbf{1} \cdot \operatorname{diox})$, and $1893402(\mathbf{1} \cdot \mathbf{D M A P})$.

Table S1. Selected structural metrics (in $\AA$ ) for $\mathbf{1} \cdot \mathbf{T H F}, \mathbf{1} \cdot \operatorname{diox}$, and $\mathbf{1} \cdot \mathbf{D M A P}$

\begin{tabular}{llll} 
& $\mathbf{1} \cdot \mathbf{T H F}$ & $\mathbf{1} \cdot \mathbf{d i o x}$ & $\mathbf{1} \cdot \mathbf{D M A P}$ \\
\hline $\mathrm{U}-\mathrm{X}(\mathrm{X}=\mathrm{O}, \mathrm{N})$ & $2.586(4)$ & $2.651(4)$ & $2.632(3)$ \\
$\mathrm{Re}-\mathrm{Cp}^{\mathrm{a}}$ & $1.759(2), 1.763(2), 1.766(2)$ & $1.759(2), 1.764(2), 1.769(2)$ & $1.769(2), 1.774(2), 1.776(2)$ \\
$\mathrm{Re}-\mathrm{N}$ & $2.032(3)-2.052(3)$ & $2.036(3)-2.049(3)$ & $2.031(4)-2.061(4)$ \\
$\mathrm{U}-\mathrm{Cp}^{\mathrm{a}}$ & $2.488(2), 2.506(2), 2.510(2)$ & $2.491(2), 2.501(2), 2.514(2)$ & $2.502(2), 2.520(2), 2.566(2)$
\end{tabular}

${ }^{\mathrm{a}} \mathrm{Cp}$ denotes the centroid of the Cp ligand. 


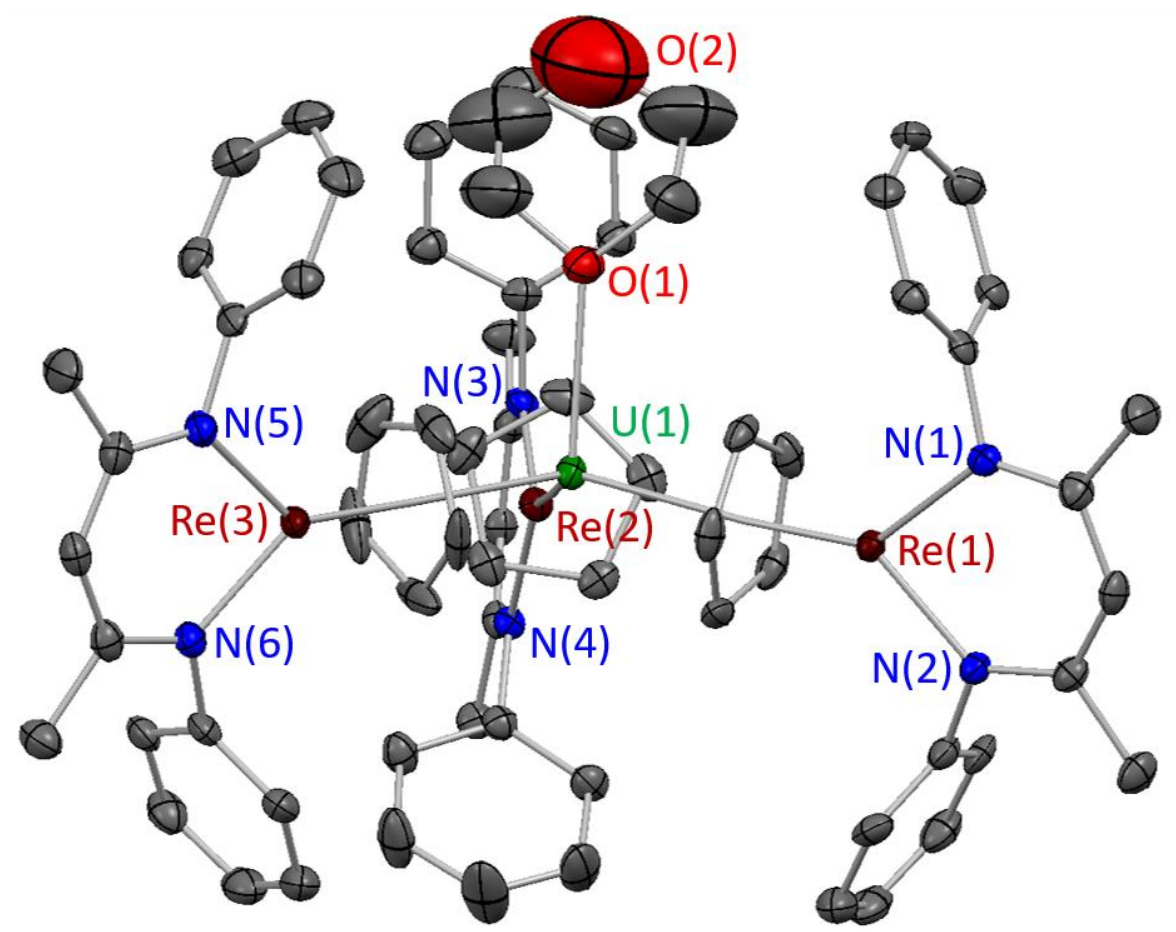

Figure S5. X-ray crystal structure of $\mathbf{1} \cdot$ diox with $50 \%$ probability ellipsoids. Hydrogen atoms, ${ }^{i}$ Pr groups, and positional disorder have been omitted for clarity. Structural metrics are shown in Table S1.

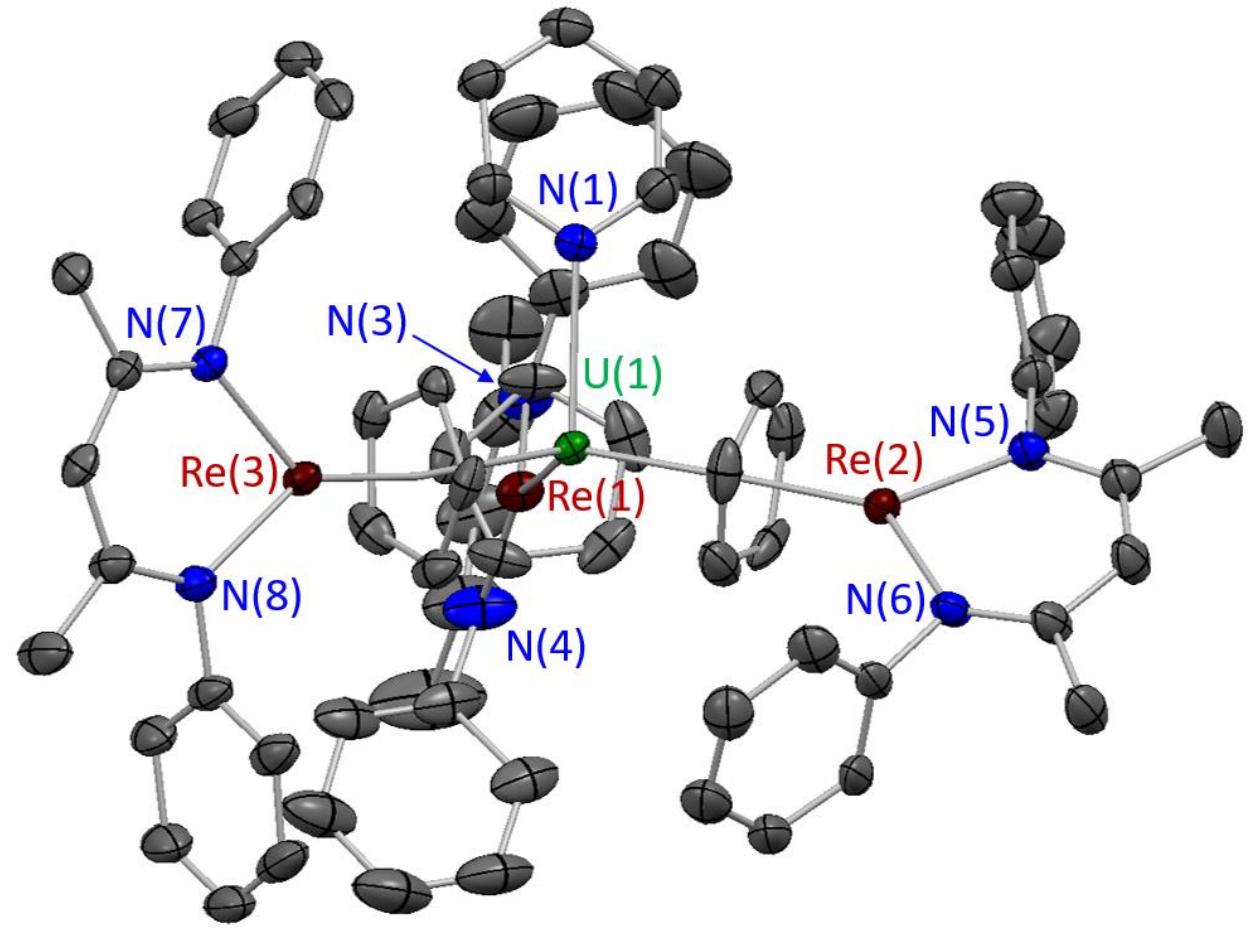

Figure S6. X-ray crystal structure of 1-DMAP with 50\% probability ellipsoids. Hydrogen atoms, ${ }^{i} \mathrm{Pr}$ groups, an $\mathrm{NMe}_{2}$ group, and positional disorder have been omitted for clarity. Structural metrics are shown in Table S1. 
Table S2. Crystallographic data for $[(\mathrm{BDI}) \mathrm{ReCp}]_{3} \mathrm{U}(\mathrm{THF})(\mathbf{1} \cdot \mathbf{T H F}) n$-hexane solvate

Identification code

Empirical formula

Formula weight

Color, habit

Temperature

Wavelength

Crystal system

Space group

Unit cell dimensions

Volume

Z

Density (calculated)

Absorption coefficient

$\mathrm{F}(000)$

Crystal size

Theta range for data collection

Index ranges

Reflections collected

Independent reflections

Completeness to theta $=25.000^{\circ}$

Absorption correction

Max. and min. transmission

Refinement method

Data / restraints / parameters

Goodness-of-fit on $\mathrm{F}^{2}$

Final R indices [I $>2 \sigma(\mathrm{I})]$

$\mathrm{R}$ indices (all data)

Extinction coefficient

Largest diff. peak and hole
1THF

$\mathrm{C}_{112} \mathrm{H}_{160} \mathrm{~N}_{6} \mathrm{ORe}_{3} \mathrm{U}$

2403.08

Red, block

100(2) K

$0.71073 \AA$

Triclinic

$\mathrm{P}-1$

$\mathrm{a}=13.1634(4) \AA \quad \alpha=98.327(2)^{\circ}$.

$\mathrm{b}=16.7458(5) \AA \quad \beta=90.663(2)^{\circ}$.

$\mathrm{c}=25.5150(8) \AA \quad \gamma=109.6710(10)^{\circ}$.
2

$1.526 \mathrm{Mg} / \mathrm{m}^{3}$

$5.054 \mathrm{~mm}^{-1}$

2398

$0.28 \times 0.18 \times 0.12 \mathrm{~mm}^{3}$

1.308 to $28.458^{\circ}$

$-17 \leq \mathrm{h} \leq 17,-22 \leq \mathrm{k} \leq 22,-34 \leq 1 \leq 33$

100568

$26080[\mathrm{R}(\mathrm{int})=0.0435]$

$100.0 \%$

Semi-empirical from equivalents

0.4920 and 0.3613

Full-matrix least-squares on $\mathrm{F}^{2}$

26080 / 4 / 1147

1.050

$\mathrm{R}_{1}=0.0345, w \mathrm{R}_{2}=0.0833$

$\mathrm{R}_{1}=0.0513, w \mathrm{R}_{2}=0.0930$

$\mathrm{n} / \mathrm{a}$

2.853 and $-1.953 \mathrm{e} \cdot \AA^{-3}$ 
Table S3. Crystallographic data for $[(\mathrm{BDI}) \mathrm{ReCp}]_{3} \mathrm{U}(1,4$-dioxane $)(\mathbf{1} \cdot \mathbf{d i o x})$ diethyl ether and $n$ hexane solvate

Identification code

Empirical formula

Formula weight

Color, habit

Temperature

Wavelength

Crystal system

Space group

Unit cell dimensions

Volume

$\mathrm{Z}$

Density (calculated)

Absorption coefficient

$\mathrm{F}(000)$

Crystal size

Theta range for data collection

Index ranges

Reflections collected

Independent reflections

Completeness to theta $=25.000^{\circ}$

Absorption correction

Max. and min. transmission

Refinement method

Data / restraints / parameters

Goodness-of-fit on $\mathrm{F}^{2}$

Final $R$ indices $[\mathrm{I}>2 \sigma(\mathrm{I})]$

$\mathrm{R}$ indices (all data)

Extinction coefficient

Largest diff. peak and hole 1diox

$\mathrm{C}_{116} \mathrm{H}_{170} \mathrm{~N}_{6} \mathrm{O}_{3} \mathrm{Re}_{3} \mathrm{U}$

2493.20

Red, plank

100(2) K

$0.71073 \AA$

Triclinic

$\mathrm{P}-1$

$\mathrm{a}=15.1739(3) \AA \quad \alpha=77.8630(10)^{\circ}$.

$\mathrm{b}=15.9697(2) \AA \quad \beta=86.540(2))^{\circ}$.

$\mathrm{c}=24.5541(4) \AA \quad \gamma=69.678(2)^{\circ}$.
2

$1.518 \mathrm{Mg} / \mathrm{m}^{3}$

$4.849 \mathrm{~mm}^{-1}$

2498

$0.14 \times 0.11 \times 0.05 \mathrm{~mm}^{3}$

3.209 to $26.372^{\circ}$

$-18 \leq \mathrm{h} \leq 18,-19 \leq \mathrm{k} \leq 19,-30 \leq 1 \leq 30$

118537

22235 [R(int) $=0.0627]$

$99.8 \%$

Semi-empirical from equivalents

1.00000 and 0.51127

Full-matrix least-squares on $\mathrm{F}^{2}$

22235 / 98 / 1234

1.034

$\mathbf{R}_{1}=0.0290, w \mathbf{R}_{2}=0.0623$

$\mathrm{R}_{1}=0.0401, w \mathrm{R}_{2}=0.0654$

$\mathrm{n} / \mathrm{a}$

1.691 and $-1.386 \mathrm{e} \cdot \AA^{-3}$ 
Table S4. Crystallographic data for $[(\mathrm{BDI}) \mathrm{ReCp}]_{3} \mathrm{U}(\mathrm{DMAP})(\mathbf{1} \cdot \mathrm{DMAP})$

Identification code

Empirical formula

Formula weight

Color, habit

Temperature

Wavelength

Crystal system

Space group

Unit cell dimensions

Volume

Z

Density (calculated)

Absorption coefficient

$\mathrm{F}(000)$

Crystal size

Theta range for data collection

Index ranges

Reflections collected

Independent reflections

Completeness to theta $=25.930^{\circ}$

Absorption correction

Max. and min. transmission

Refinement method

Data / restraints / parameters

Goodness-of-fit on $\mathrm{F}^{2}$

Final R indices [I $>2 \sigma(\mathrm{I})]$

$\mathrm{R}$ indices (all data)

Extinction coefficient

Largest diff. peak and hole
1DMAP

$\mathrm{C}_{109} \mathrm{H}_{148} \mathrm{~N}_{8} \mathrm{Re}_{3} \mathrm{U}$

2366.98

Red, block

100(2) K

$0.7288 \AA$

Monoclinic

P $21 / n$

$\mathrm{a}=16.8272(8) \AA \quad \alpha=90^{\circ}$.

$\mathrm{b}=19.4767(9) \AA \quad \beta=98.321(2)^{\circ}$.

$\mathrm{c}=30.7821(15) \AA \quad \gamma=90^{\circ}$.

9982.3(8) $\AA^{3}$

4

$1.575 \mathrm{Mg} / \mathrm{m}^{3}$

$4.630 \mathrm{~mm}^{-1}$

4700

$0.06 \times 0.035 \times 0.025 \mathrm{~mm}^{3}$

2.026 to $26.042^{\circ}$

$-20 \leq \mathrm{h} \leq 20,-23 \leq \mathrm{k} \leq 23,-37 \leq 1 \leq 37$

128667

$18120[\mathrm{R}(\mathrm{int})=0.0651]$

$99.2 \%$

Semi-empirical from equivalents

0.7457 and 0.6178

Full-matrix least-squares on $\mathrm{F}^{2}$

18120 / $13 / 1151$

1.026

$\mathrm{R}_{1}=0.0289, w \mathrm{R}_{2}=0.0726$

$\mathrm{R}_{1}=0.0320, w \mathrm{R}_{2}=0.0743$

$\mathrm{n} / \mathrm{a}$

2.134 and $-1.460 \mathrm{e} \cdot \AA^{-3}$ 


\section{EPR spectroscopy}

EPR spectroscopy was performed in the Cal-EPR center in Department of Chemistry, University of California at Davis. CW-EPR experiments were performed on the Bruker Biospin EleXsys E500 spectrometer with a super high Q resonator (ER4122SHQE) in perpendicular mode. Cryogenic temperatures were achieved by using an ESR900 liquid helium cryostat with a temperature controller (Oxford Instrument ITC503). Spectrometer settings were: conversion time, $40 \mathrm{~ms}$; modulation amplitude, $0.5 \mathrm{mT}$; modulation frequency, $100 \mathrm{kHz}$.

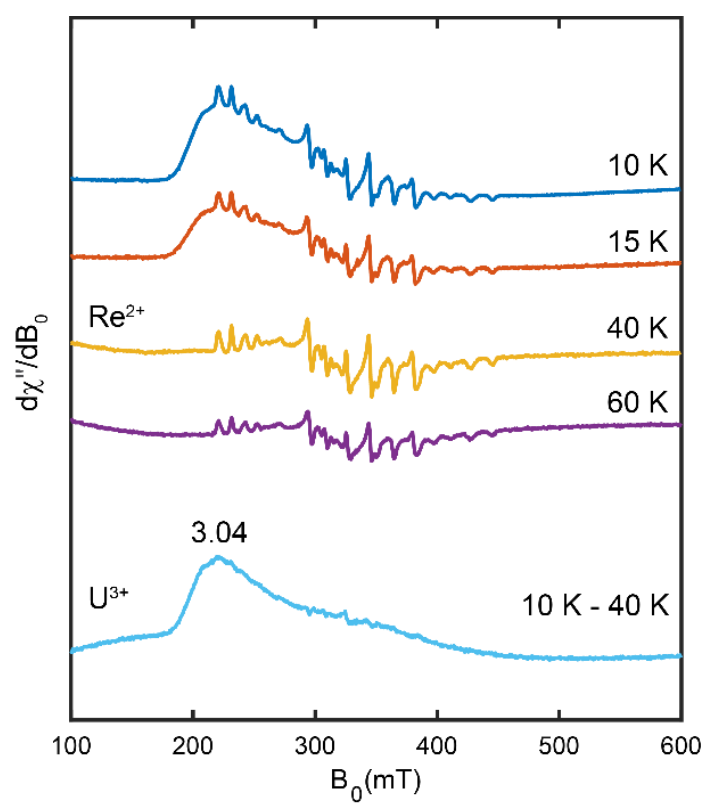

Figure S7. EPR spectra of $\mathbf{1} \cdot \mathbf{T H F}$ recorded at different temperatures to distinguish the contributions from 1. THF and the $\operatorname{Re}\left(\eta^{5}-\mathrm{Cp}\right)(\mathrm{BDI})$ impurity. ${ }^{15}$ The EPR spectrum of $\mathbf{1} \cdot \mathbf{T H F}$ at the bottom in light blue was obtained by spectral subtraction. Note that the $5 d$ electrons in $\operatorname{Re}\left(\eta^{5}-\right.$ Cp)(BDI) relax much slower than the $5 f$ electrons in 1·THF. Therefore, the relative intensity of their EPR signals does not reflect their actual ratio in the sample. 


\section{Computational details and coordinates of optimized structures}

Calculations were carried out with Gaussian 09 program ${ }^{16}$ with B3PW91-GD3BJ, ${ }^{17-19}$ which includes dispersion effects according to the Grimme's scheme. The uranium and rhenium atoms were treated with the corresponding Stuttgart-Dresden RECP (relativistic effective core potential) in combination with its adapted basis sets ${ }^{20-22}$ and augmented by an extra polarization function. ${ }^{23}$ For all the remaining atoms, the $6-31 \mathrm{G}(\mathrm{d}, \mathrm{p})$ basis set was used. ${ }^{24}$ Geometry optimizations were carried out without any symmetry restrictions. Natural population analysis (NPA) was performed using Weinhold's methodology. ${ }^{25,26}$ Finally, Chemcraft was used for the visualization of the molecular orbitals. ${ }^{27}$

Table S5. Comparison of bond lengths (in $\AA$ ) in calculated structures (Calc) versus X-ray crystal structures (X-ray).

\begin{tabular}{l|ll|ll} 
& $\begin{array}{l}\mathbf{1} \cdot \mathbf{T H F} \\
(\mathrm{Calc})\end{array}$ & $\begin{array}{l}\mathbf{1} \cdot \mathbf{T H F} \\
(\mathrm{X}-\mathrm{ray})\end{array}$ & $\begin{array}{l}{\left[\mathbf{R e}\left(\boldsymbol{\eta}^{5}-\mathbf{C p}\right)(\mathbf{B D I})\right]^{-}} \\
(\mathrm{Calc})\end{array}$ & $\begin{array}{l}\mathbf{N a}\left[\mathbf{R e}\left(\boldsymbol{\eta}^{5}-\mathbf{C p}\right)(\mathbf{B D I})\right] \\
(\mathrm{X}-\mathrm{ray})\end{array}$ \\
\hline $\mathrm{U}-\mathrm{O}$ & 2.547 & $2.586(4)$ & - & - \\
$\mathrm{Re}-\mathrm{Cp}^{\mathrm{a}}$ & $1.765,1.766,1.771$ & $1.759(2), 1.763(2), 1.766(2)$ & 1.857 & $1.816(2)$ \\
$\mathrm{Re}-\mathrm{N}$ & $2.039-2.043$ & $2.032(3)-2.052(3)$ & 2.035 & $2.031(3)$ \\
$\mathrm{U}-\mathrm{Cp}^{\mathrm{a}}$ & $2.420,2.445,2.454$ & $2.488(2), 2.506(2), 2.510(2)$ & - & -
\end{tabular}

${ }^{\mathrm{a}} \mathrm{Cp}$ denotes the centroid of the Cp ligand. 


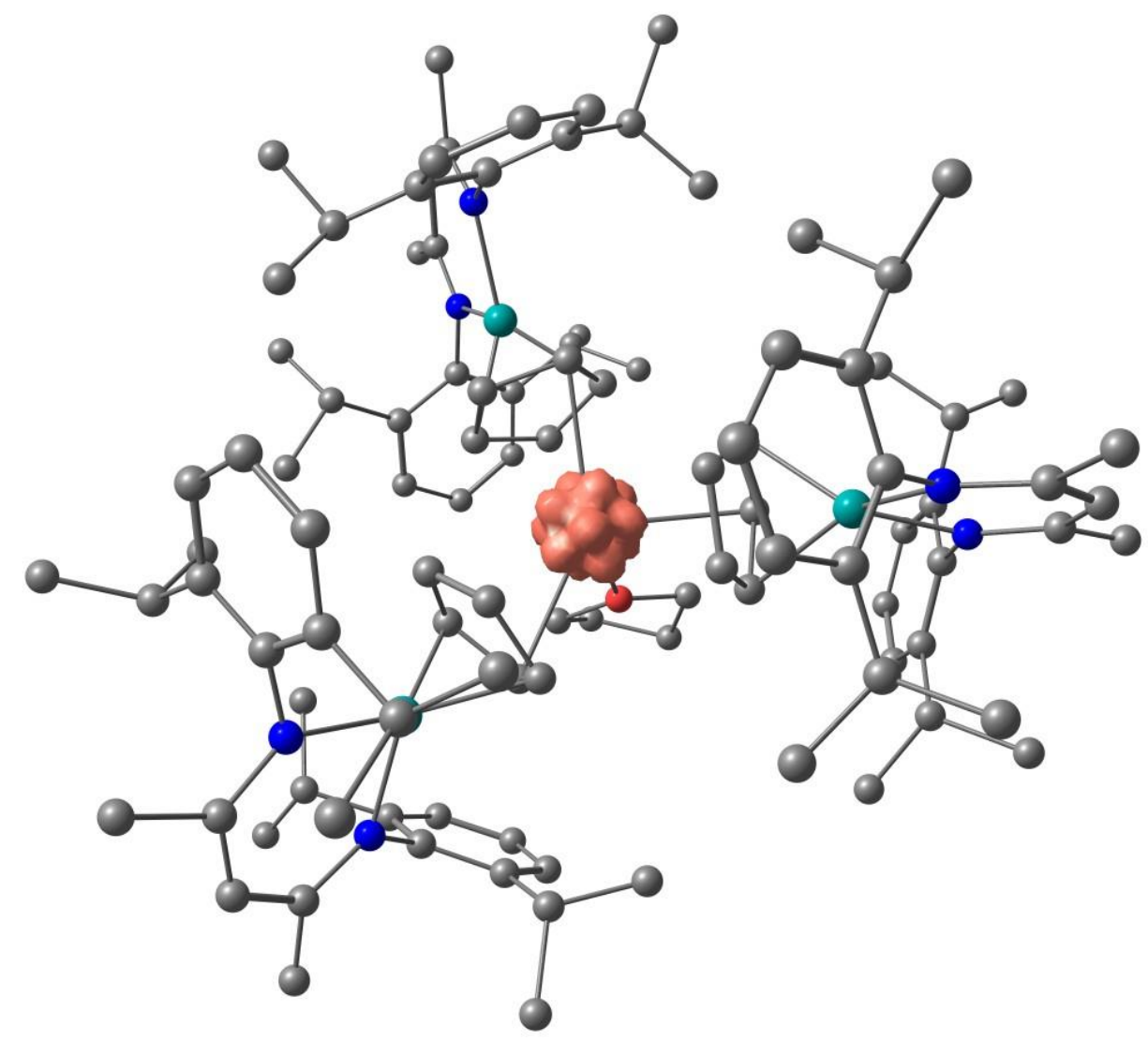

Figure S8. Unpaired spin density plot of 1 THF demonstrating localization of spin density to the uranium center.

Table S6. Coordinates for optimized structure of $\left[\operatorname{Re}\left(\eta^{5}-\mathrm{Cp}\right)(\mathrm{BDI})\right]^{-}$

$\begin{array}{cccc}6 & 8.141298000 & 1.139072000 & 4.832697000 \\ 1 & 8.457802000 & 2.172219000 & 4.887541000 \\ 6 & 8.928481000 & 0.048214000 & 4.353832000 \\ 1 & 9.930282000 & 0.125232000 & 3.951052000 \\ 6 & 8.132190000 & -1.152709000 & 4.406421000 \\ 1 & 8.441272000 & -2.138949000 & 4.085898000 \\ 6 & 6.857813000 & -0.822297000 & 4.938780000 \\ 1 & 6.046999000 & -1.518886000 & 5.102869000 \\ 6 & 6.878453000 & 0.586015000 & 5.279035000 \\ 1 & 6.033315000 & 1.156914000 & 5.645443000 \\ 6 & 7.439117000 & -2.891758000 & 7.426007000 \\ 6 & 8.136255000 & -4.041582000 & 6.995813000 \\ 6 & 10.289142000 & -3.762589000 & 5.716835000\end{array}$




$\begin{array}{cccc}1 & 9.948028000 & -4.451480000 & 4.934318000 \\ 1 & 11.382665000 & -3.824640000 & 5.776664000 \\ 1 & 10.012784000 & -2.740437000 & 5.442024000 \\ 6 & 9.652446000 & -4.106356000 & 7.066549000 \\ 1 & 9.978218000 & -3.331529000 & 7.766569000 \\ 6 & 10.159195000 & -5.454728000 & 7.585715000 \\ 1 & 9.676568000 & -5.730229000 & 8.529599000 \\ 1 & 11.241636000 & -5.414038000 & 7.752428000 \\ 1 & 9.971885000 & -6.263385000 & 6.869583000 \\ 6 & 7.406668000 & -5.113371000 & 6.476010000 \\ 1 & 7.936168000 & -5.994511000 & 6.121461000 \\ 6 & 6.017939000 & -5.077141000 & 6.411871000 \\ 1 & 5.464687000 & -5.922559000 & 6.009992000 \\ 6 & 5.339811000 & -3.947631000 & 6.866786000 \\ 1 & 4.255004000 & -3.922768000 & 6.813723000 \\ 6 & 6.024162000 & -2.844333000 & 7.378242000 \\ 6 & 3.922331000 & -1.416199000 & 7.178903000 \\ 1 & 3.983209000 & -1.494878000 & 6.089162000 \\ 1 & 3.523739000 & -0.425602000 & 7.423452000 \\ 1 & 3.191160000 & -2.155113000 & 7.532158000 \\ 6 & 5.289765000 & -1.596873000 & 7.836343000 \\ 1 & 5.931603000 & -0.755208000 & 7.532488000 \\ 6 & 5.150789000 & -1.526005000 & 9.361349000 \\ 1 & 4.611205000 & -2.398180000 & 9.752227000 \\ 1 & 4.593517000 & -0.626346000 & 9.649704000 \\ 1 & 6.129456000 & -1.473801000 & 9.840920000 \\ 6 & 8.303230000 & -3.043847000 & 10.040335000 \\ 1 & 7.262177000 & -3.367024000 & 9.951515000 \\ 1 & 8.511716000 & -2.831291000 & 11.092595000 \\ 1 & 8.919203000 & -3.902263000 & 9.745069000 \\ 6 & 8.599606000 & -1.826099000 & 9.196475000 \\ 6 & 9.351261000 & -0.801054000 & 9.772833000 \\ 1 & 9.633801000 & -0.938397000 & 10.812605000 \\ 6 & 9.798777000 & 0.370520000 & 9.161784000 \\ 6 & 10.662048000 & 1.303702000 & 9.979174000 \\ 1 & 11.720546000 & 1.231676000 & 9.698836000 \\ 1 & 10.582210000 & 1.054779000 & 11.041050000 \\ 1 & 10.380718000 & 2.351928000 & 9.845812000 \\ 6 & 10.089709000 & 1.862595000 & 7.346448000 \\ & 1.439447000 & 1.865736000 & 6.933362000\end{array}$




$\begin{array}{lccc}6 & 13.716562000 & 0.905303000 & 7.517779000 \\ 1 & 14.293706000 & 1.444254000 & 6.757387000 \\ 1 & 14.247653000 & -0.031440000 & 7.721774000 \\ 1 & 13.725060000 & 1.510266000 & 8.431086000 \\ 6 & 12.289512000 & 0.612483000 & 7.048339000 \\ 1 & 11.816549000 & -0.035267000 & 7.792420000 \\ 6 & 12.288486000 & -0.174349000 & 5.734796000 \\ 1 & 11.260570000 & -0.472099000 & 5.503822000 \\ 1 & 12.905065000 & -1.077421000 & 5.821498000 \\ 1 & 12.682196000 & 0.435107000 & 4.912083000 \\ 6 & 11.969350000 & 3.034513000 & 6.380868000 \\ 1 & 13.001293000 & 3.040726000 & 6.038383000 \\ 6 & 11.205162000 & 4.190798000 & 6.268216000 \\ 1 & 11.635335000 & 5.093812000 & 5.841717000 \\ 6 & 9.881378000 & 4.182422000 & 6.704635000 \\ 1 & 9.285271000 & 5.086212000 & 6.612992000 \\ 6 & 9.301285000 & 3.034535000 & 7.244958000 \\ 6 & 7.697948000 & 3.221378000 & 9.193799000 \\ 1 & 8.186673000 & 2.418493000 & 9.74834000 \\ 1 & 6.637669000 & 3.219717000 & 9.474817000 \\ 1 & 8.130709000 & 4.179931000 & 9.507755000 \\ 6 & 7.848906000 & 3.011969000 & 7.683448000 \\ 1 & 7.494960000 & 1.994175000 & 7.459807000 \\ 6 & 6.966726000 & 3.997038000 & 6.918221000 \\ 1 & 7.183157000 & 5.041432000 & 7.178597000 \\ 1 & 5.913578000 & 3.816431000 & 7.160721000 \\ 1 & 7.087614000 & 3.884186000 & 5.836726000 \\ 7 & 8.167499000 & -1.768839000 & 7.912624000 \\ 7 & 9.517009000 & 0.666681000 & 7.867746000 \\ 75 & 8.410910000 & -0.338268000 & 6.485999000\end{array}$

Table S7. Coordinates for optimized structure of $\mathbf{1} \cdot \mathbf{T H F}$

$\begin{array}{llll}6 & 8.416345000 & 4.995140000 & 19.312717000 \\ 1 & 9.407617000 & 5.189773000 & 19.695107000 \\ 6 & 7.203933000 & 4.747182000 & 20.113930000 \\ 1 & 7.202553000 & 4.521116000 & 21.172440000 \\ 6 & 6.088708000 & 4.476283000 & 19.186801000 \\ 1 & 5.071932000 & 4.225607000 & 19.452077000\end{array}$




$\begin{array}{cccc}6 & 6.554906000 & 4.822820000 & 17.856045000 \\ 1 & 5.960788000 & 4.769056000 & 16.956672000 \\ 6 & 7.987177000 & 5.138562000 & 17.931276000 \\ 1 & 8.631537000 & 5.354177000 & 17.092548000 \\ 6 & 7.732715000 & 0.264701000 & 20.200344000 \\ 1 & 8.655577000 & -0.295852000 & 20.136041000 \\ 6 & 7.502490000 & 1.435062000 & 21.044104000 \\ 1 & 8.151280000 & 1.788206000 & 21.831238000 \\ 6 & 6.105766000 & 1.849391000 & 20.817481000 \\ 1 & 5.644665000 & 2.727791000 & 21.244708000 \\ 6 & 5.612093000 & 1.148438000 & 19.622025000 \\ 1 & 4.644869000 & 1.271544000 & 19.155114000 \\ 6 & 6.555267000 & 0.087028000 & 19.340100000 \\ 1 & 6.445376000 & -0.641371000 & 18.552346000 \\ 6 & 9.361778000 & 1.321182000 & 16.833571000 \\ 1 & 10.321226000 & 0.998985000 & 17.207600000 \\ 6 & 9.058283000 & 2.610537000 & 16.209612000 \\ 1 & 9.727474000 & 3.458080000 & 16.171778000 \\ 6 & 7.625460000 & 2.630844000 & 15.875114000 \\ 1 & 7.073315000 & 3.458740000 & 15.458406000 \\ 6 & 7.065230000 & 1.346843000 & 16.218201000 \\ 1 & 6.036451000 & 1.055151000 & 16.068423000 \\ 6 & 8.165879000 & 0.485778000 & 16.671463000 \\ 1 & 8.057758000 & -0.520643000 & 17.047997000 \\ 6 & 7.499937000 & 9.551548000 & 19.305647000 \\ 6 & 6.142924000 & 9.881878000 & 19.353988000 \\ 1 & 5.912304000 & 10.933103000 & 19.487419000 \\ 6 & 5.047304000 & 9.019617000 & 19.246634000 \\ 6 & 8.502231000 & 10.665322000 & 19.478018000 \\ 1 & 8.810644000 & 10.734855000 & 20.527641000 \\ 1 & 8.067573000 & 11.626305000 & 19.194847000 \\ 1 & 9.406390000 & 10.496317000 & 18.890216000 \\ 6 & 3.668353000 & 9.617894000 & 19.367714000 \\ 1 & 2.950860000 & 9.127490000 & 18.707013000 \\ 1 & 3.687293000 & 10.686468000 & 19.143959000 \\ 1 & 3.295147000 & 9.495505000 & 20.390839000 \\ 6 & 9.332630000 & 8.022822000 & 19.036208000 \\ 1 & 10.122104000 & 7.851401000 & 20.189423000 \\ & 11.448810000 & 7.437931000 & 20.037132000 \\ & 12.064568000 & 7.294559000 & 20.921664000\end{array}$




$\begin{array}{cccc}6 & 11.991994000 & 7.210167000 & 18.777660000 \\ 1 & 13.024850000 & 6.888966000 & 18.675970000 \\ 6 & 11.205829000 & 7.407678000 & 17.644775000 \\ 1 & 11.636988000 & 7.234845000 & 16.663884000 \\ 6 & 9.876042000 & 7.823457000 & 17.743857000 \\ 6 & 9.553105000 & 8.060518000 & 21.579739000 \\ 1 & 8.551484000 & 8.484578000 & 21.465513000 \\ 6 & 10.400662000 & 9.038868000 & 22.399236000 \\ 1 & 11.389782000 & 8.624499000 & 22.623100000 \\ 1 & 9.913762000 & 9.258674000 & 23.355186000 \\ 1 & 10.552880000 & 9.982360000 & 21.866479000 \\ 6 & 9.387685000 & 6.728298000 & 22.319284000 \\ 1 & 8.678884000 & 6.090579000 & 21.788151000 \\ 1 & 9.003584000 & 6.893615000 & 23.331919000 \\ 1 & 10.345115000 & 6.200621000 & 22.403696000 \\ 6 & 9.028238000 & 8.027568000 & 16.499854000 \\ 1 & 8.021915000 & 7.672960000 & 16.759571000 \\ 6 & 8.910173000 & 9.509351000 & 16.121316000 \\ 1 & 8.408706000 & 10.085563000 & 16.900154000 \\ 1 & 8.324897000 & 9.618550000 & 15.201794000 \\ 1 & 9.899347000 & 9.948131000 & 15.947948000 \\ 6 & 9.512642000 & 7.229001000 & 15.288506000 \\ 1 & 10.444040000 & 7.633727000 & 14.876027000 \\ 1 & 8.762934000 & 7.269536000 & 14.493496000 \\ 1 & 9.690133000 & 6.174424000 & 15.522325000 \\ 6 & 4.015221000 & 6.884614000 & 18.880904000 \\ 6 & 3.667111000 & 6.522628000 & 17.557647000 \\ 6 & 2.619159000 & 5.617622000 & 17.376769000 \\ 1 & 2.345975000 & 5.314086000 & 16.371256000 \\ 6 & 1.925348000 & 5.084179000 & 18.459988000 \\ 1 & 1.119306000 & 4.374711000 & 18.295614000 \\ 6 & 2.269459000 & 5.466392000 & 19.750713000 \\ 1 & 1.730623000 & 5.047050000 & 20.595758000 \\ 6 & 3.305901000 & 6.374652000 & 19.984533000 \\ 6 & 4.432486000 & 7.078559000 & 16.368216000 \\ 1 & 5.477244000 & 7.163013000 & 16.694406000 \\ 6 & 3.957312000 & 8.484803000 & 15.981757000 \\ 1 & 2.888467000 & 8.482527000 & 15.739241000 \\ 1 & 4.503730000 & 8.840539000 & 15.101203000 \\ 1 & 4.126970000 & 9.202739000 & 16.785554000\end{array}$




\begin{tabular}{|c|c|c|c|}
\hline & 4.396314000 & 6.170517000 & 15.137527000 \\
\hline & 4.643973000 & 5.130184000 & 15.368656000 \\
\hline & 5.115222000 & 6.528076000 & 14.393531000 \\
\hline & 3.410905000 & 6.171131000 & 14.657459000 \\
\hline & 3.667541000 & 6.745444000 & 21.410497000 \\
\hline & 4.430943000 & 7.527866000 & 21.367875000 \\
\hline & 2.459037000 & 7.292136000 & 22.178114000 \\
\hline & 1.697855000 & 6.519093000 & 22.330439000 \\
\hline & 1.982345000 & 0000 & 86000 \\
\hline & 2000 & 000 & 000 \\
\hline & 39000 & 5.556 & 22.152363000 \\
\hline & 4.527547000 & 5.826 & 5608000 \\
\hline & 0000 & 5.24 & 73000 \\
\hline & 3.60 & & 000 \\
\hline & 3.99 & & 000 \\
\hline & $4.75 \mathrm{c}$ & -2.3 & 1000 \\
\hline & 4.33477 & -3.0094 & 000 \\
\hline & 6.00459 & -1.7971 & 281000 \\
\hline & 2.6619 & -2.86 & 16000 \\
\hline & 1.857176000 & -2.1644 & 460000 \\
\hline & 2.615 & -3.69 & 44000 \\
\hline & 2.453 & 71000 & 225000 \\
\hline & 6.616 & -2.12 & 97000 \\
\hline & 7.70 & -2.2 & 7000 \\
\hline & 6.19201 & -3.047926000 & 50000 \\
\hline & 6.413257000 & $-1.3217 \varepsilon$ & 79000 \\
\hline & 3.6037 & 4000 & 94000 \\
\hline & 3.9908 & -2.158148000 & 2236000 \\
\hline & 3.27703 & -2.049191000 & 18.057568000 \\
\hline & 3.554721000 & -2.670697000 & 17.213418000 \\
\hline & 2.224539000 & -1.148201000 & 17.920411000 \\
\hline & 1.693594000 & -1.071799000 & 16.975944000 \\
\hline & 1.859308000 & -0.350048000 & 18.997598000 \\
\hline & 1.037881000 & 0.353417000 & 18.890268000 \\
\hline & 2.525997000 & -0.438282000 & 20.222855000 \\
\hline & 5.163077000 & -3.118245000 & 19.375636000 \\
\hline & 5.882177000 & -2.637598000 & 20.051483000 \\
\hline & 5.879266000 & -3.377043000 & 18.048251000 \\
\hline & 5.277907000 & -3.992918000 & 17.369809000 \\
\hline & 6.814185000 & -3.916946000 & 18.229994000 \\
\hline
\end{tabular}




$\begin{array}{cccc}1 & 6.120065000 & -2.448746000 & 17.521311000 \\ 6 & 4.752595000 & -4.451811000 & 20.011115000 \\ 1 & 4.383615000 & -4.313894000 & 21.028733000 \\ 1 & 5.611328000 & -5.130618000 & 20.059502000 \\ 1 & 3.967475000 & -4.939502000 & 19.422127000 \\ 6 & 2.094692000 & 0.444491000 & 21.379466000 \\ 1 & 2.661220000 & 0.134074000 & 22.262376000 \\ 6 & 2.438005000 & 1.915465000 & 21.121557000 \\ 1 & 3.517210000 & 2.045292000 & 21.034133000 \\ 1 & 2.086907000 & 2.542577000 & 21.948711000 \\ 1 & 1.971842000 & 2.280299000 & 20.200255000 \\ 6 & 0.600928000 & 0.286464000 & 21.684741000 \\ 1 & -0.017214000 & 0.676580000 & 20.868908000 \\ 1 & 0.335043000 & 0.840453000 & 22.591299000 \\ 1 & 0.326275000 & -0.762626000 & 21.830822000 \\ 6 & 7.953753000 & -0.494742000 & 23.510854000 \\ 6 & 9.069087000 & -1.149699000 & 22.935348000 \\ 6 & 10.339890000 & -0.621089000 & 23.173531000 \\ 1 & 11.207842000 & -1.106678000 & 22.738457000 \\ 6 & 10.517898000 & 0.520956000 & 23.951748000 \\ 1 & 11.515928000 & 0.915661000 & 24.121309000 \\ 6 & 9.411271000 & 1.144650000 & 24.515907000 \\ 1 & 9.548102000 & 2.033043000 & 25.126897000 \\ 6 & 8.120146000 & 0.644105000 & 24.322280000 \\ 6 & 8.884390000 & -2.376215000 & 22.058181000 \\ 1 & 7.951817000 & -2.209564000 & 21.503278000 \\ 6 & 8.705869000 & -3.658113000 & 22.880799000 \\ 1 & 9.568105000 & -3.826331000 & 23.536110000 \\ 1 & 8.611773000 & -4.523613000 & 22.215813000 \\ 1 & 7.806156000 & -3.618159000 & 23.496335000 \\ 6 & 10.010453000 & -2.571339000 & 21.041375000 \\ 1 & 10.239561000 & -1.651781000 & 20.493394000 \\ 1 & 9.724402000 & -3.333375000 & 20.310305000 \\ 1 & 10.937387000 & -2.911666000 & 21.517273000 \\ 6 & 6.938213000 & 1.351749000 & 24.958695000 \\ 1 & 6.051176000 & 0.736972000 & 24.781968000 \\ 6 & 7.121881000 & 1.508224000 & 26.472202000 \\ 1 & 7.354646000 & 0.552400000 & 26.951253000 \\ 1 & 6.210253000 & 1.908126000 & 26.928254000 \\ 1 & 7.936994000 & 2.200990000 & 26.707772000\end{array}$




$\begin{array}{cccc}6 & 6.677650000 & 2.711136000 & 24.300917000 \\ 1 & 7.556997000 & 3.361839000 & 24.370262000 \\ 1 & 5.840938000 & 3.222802000 & 24.788537000 \\ 1 & 6.422577000 & 2.578161000 & 23.249143000 \\ 6 & 10.596037000 & -0.032066000 & 12.595530000 \\ 6 & 9.588434000 & -0.006781000 & 11.629830000 \\ 1 & 9.859029000 & -0.379854000 & 10.647974000 \\ 6 & 8.289412000 & 0.495703000 & 11.760696000 \\ 6 & 11.959808000 & -0.514058000 & 12.168737000 \\ 1 & 12.583726000 & 0.328943000 & 11.851411000 \\ 1 & 11.875537000 & -1.199924000 & 11.322921000 \\ 1 & 12.491024000 & -1.013595000 & 12.980846000 \\ 6 & 7.442448000 & 0.569602000 & 10.514677000 \\ 1 & 6.380796000 & 0.440465000 & 10.730288000 \\ 1 & 7.755094000 & -0.186828000 & 9.791355000 \\ 1 & 7.554699000 & 1.550817000 & 10.039222000 \\ 6 & 11.528732000 & 0.475193000 & 14.743097000 \\ 6 & 12.391396000 & 1.588131000 & 14.677688000 \\ 6 & 13.374979000 & 1.729920000 & 15.661803000 \\ 1 & 14.030316000 & 2.596718000 & 15.634630000 \\ 6 & 13.535956000 & 0.780820000 & 16.664746000 \\ 1 & 14.312617000 & 0.901510000 & 17.415074000 \\ 6 & 12.702390000 & -0.335557000 & 16.691324000 \\ 1 & 12.834325000 & -1.079014000 & 17.471720000 \\ 6 & 11.690196000 & -0.509367000 & 15.745481000 \\ 6 & 12.271606000 & 2.639286000 & 13.587717000 \\ 1 & 11.549284000 & 2.278372000 & 12.850519000 \\ 6 & 11.721238000 & 3.961158000 & 14.132191000 \\ 1 & 12.338766000 & 4.341132000 & 14.954170000 \\ 1 & 10.702834000 & 3.815267000 & 14.494164000 \\ 1 & 11.696016000 & 4.724366000 & 13.347035000 \\ 6 & 13.609143000 & 2.863494000 & 12.872929000 \\ 1 & 14.342765000 & 3.339589000 & 13.532445000 \\ 1 & 13.473065000 & 3.520227000 & 12.007329000 \\ 1 & 14.043697000 & 1.921497000 & 12.524973000 \\ 6 & 10.755381000 & -1.702085000 & 15.823395000 \\ 1 & 9.807906000 & -1.378921000 & 15.375779000 \\ 6 & 10.459254000 & -2.141451000 & 17.259377000 \\ 1 & 11.321845000 & -2.623222000 & 17.733053000 \\ 1 & 9.641726000 & -2.869894000 & 17.266074000\end{array}$




\begin{tabular}{lccc}
1 & 10.162793000 & -1.296395000 & 17.887672000 \\
6 & 11.280871000 & -2.883257000 & 15.000275000 \\
1 & 11.373050000 & -2.621714000 & 13.943665000 \\
1 & 10.597146000 & -3.736178000 & 15.073540000 \\
1 & 12.265353000 & -3.204729000 & 15.359397000 \\
6 & 6.483611000 & 1.505863000 & 12.967350000 \\
6 & 5.426640000 & 0.722329000 & 13.483295000 \\
6 & 4.181483000 & 1.330506000 & 13.656706000 \\
1 & 3.359198000 & 0.753316000 & 14.067284000 \\
6 & 3.977665000 & 2.668935000 & 13.329561000 \\
1 & 3.004616000 & 3.126095000 & 13.484654000 \\
6 & 5.023343000 & 3.415204000 & 12.799058000 \\
1 & 4.859385000 & 4.457311000 & 12.540232000 \\
6 & 6.285405000 & 2.849587000 & 12.596611000 \\
6 & 7.409167000 & 3.691841000 & 12.018152000 \\
1 & 8.236020000 & 3.020315000 & 11.770555000 \\
6 & 7.951414000 & 4.694278000 & 13.042077000 \\
1 & 7.154762000 & 5.345723000 & 13.418419000 \\
1 & 8.723968000 & 5.326981000 & 12.592187000 \\
1 & 8.397649000 & 4.163567000 & 13.885641000 \\
6 & 6.978978000 & 4.406924000 & 10.733164000 \\
1 & 6.549919000 & 3.708049000 & 10.008437000 \\
1 & 7.837152000 & 4.902813000 & 10.267237000 \\
1 & 6.226385000 & 5.176892000 & 10.933781000 \\
6 & 5.658936000 & -0.728107000 & 13.869090000 \\
1 & 6.672781000 & -0.772185000 & 14.290880000 \\
6 & 4.689912000 & -1.242794000 & 14.931851000 \\
1 & 4.647998000 & -0.598411000 & 15.814738000 \\
1 & 5.004832000 & -2.236998000 & 15.262046000 \\
1 & 3.668748000 & -1.340813000 & 14.545499000 \\
6 & 5.628398000 & -1.657848000 & 12.649804000 \\
1 & 4.668328000 & -1.583937000 & 12.126431000 \\
1 & 5.764465000 & -2.698914000 & 12.963040000 \\
1 & 6.424931000 & -1.420133000 & 11.943354000 \\
7 & 7.931572000 & 8.286237000 & 19.169010000 \\
7 & 5.181067000 & 7.691248000 & 19.093429000 \\
7 & 4.385556000 & -1.368622000 & 21.535792000 \\
\hline & 10.437776000 & -0.947960000 & 23.175529000 \\
\hline
\end{tabular}




$\begin{array}{lrrr}8 & 10.173497000 & 2.517174000 & 19.482164000 \\ 75 & 6.869214000 & 6.546945000 & 19.085465000 \\ 75 & 6.019463000 & -0.151467000 & 21.398143000 \\ 75 & 8.718265000 & 1.121264000 & 14.745514000 \\ 92 & 7.777743000 & 2.488829000 & 18.618307000 \\ 6 & 10.601753000 & 2.318458000 & 20.851082000 \\ 1 & 10.296995000 & 1.319580000 & 21.163671000 \\ 1 & 10.103215000 & 3.060178000 & 21.486647000 \\ 6 & 12.103619000 & 2.529546000 & 20.821260000 \\ 1 & 12.608184000 & 1.616451000 & 20.487497000 \\ 1 & 12.498329000 & 2.805094000 & 21.801929000 \\ 6 & 12.239058000 & 3.633617000 & 19.773809000 \\ 1 & 11.977100000 & 4.607474000 & 20.199588000 \\ 1 & 13.239497000 & 3.707415000 & 19.341074000 \\ 6 & 11.208064000 & 3.219751000 & 18.737042000 \\ 1 & 10.752346000 & 4.071576000 & 18.226659000 \\ 1 & 11.620485000 & 2.528115000 & 17.996763000\end{array}$




\section{References}

(1) Monreal, M. J.; Thomson, R. K.; Cantat, T.; Travia, N. E.; Scott, B. L.; Kiplinger, J. L. $\mathrm{UI}_{4}(1,4 \text {-dioxane })_{2}$, $\left[\mathrm{UCl}_{4}(1,4 \text {-dioxane })\right]_{2}$, and $\mathrm{UI}_{3}(1,4 \text {-dioxane })_{1.5}$ : Stable and Versatile Starting Materials for Low- and High-valent Uranium Chemistry. Organometallics 2011, 30, 2031.

(2) Carmichael, C. D.; Jones, N. A.; Arnold, P. L. Low-Valent Uranium Iodides: Straightforward Solution Syntheses of $\mathrm{UI}_{3}$ and $\mathrm{UI}_{4}$ Etherates. Inorg. Chem. 2008, 47, 8577.

(3) Kiplinger, J. L.; Morris, D. E.; Scott, B. L.; Burns, C. J. Convenient Synthesis, Structure, and Reactivity of $\left(\mathrm{C}_{5} \mathrm{Me}_{5}\right) \mathrm{U}\left(\mathrm{CH}_{2} \mathrm{C}_{6} \mathrm{H}_{5}\right)_{3}$ : A Simple Strategy for the Preparation of Monopentamethylcyclopentadienyl Uranium(IV) Complexes. Organometallics 2002, 21, 5978.

(4) Araya, M. A.; Cotton, F. A.; Matonic, J. H.; Murillo, C. A. An Efficient Reduction Process Leading to Titanium(II) and Niobium(II): Preparation and Structural Characterization of trans$\mathrm{MCl}_{2}(\mathrm{py})_{4}$ Compounds, $\mathrm{M}=\mathrm{Ti}, \mathrm{Nb}$, and Mn. Inorg. Chem. 1995, 34, 5424.

(5) Lawrence, E. J.; Oganesyan, V. S.; Wildgoose, G. G.; Ashley, A. E. Exploring the fate of the tris(pentafluorophenyl)borane radical anion in weakly coordinating solvents. Dalton Trans. 2013, 42, 782.

(6) Lohrey, T. D.; Maron, L.; Bergman, R. G.; Arnold, J. Heterotetrametallic Re-Zn-Zn-Re Complex Generated by an Anionic Rhenium(I) $\beta$-Diketiminate. J. Am. Chem. Soc. 2019, 141, 800 .

(7) Bruker. APEX2, APEX3, and SAINT. Bruker AXS Inc., Madison, Wisconsin, USA.

(8) Bruker. SADABS. Bruker AXS Inc., Madison, Wisconsin, USA.

(9) Rigaku Oxford Diffraction, (2015), CrysAlisPro Software system, version 1.171.39.7a, Rigaku Corporation, Oxford, UK.

(10) Sheldrick, G. M. SHELXT - Integrated space-group and crystal-structure determination. Acta. Cryst. 2015, A71, 3.

(11) Sheldrick, G. M. A short history of SHELX. Acta Cryst. 2008, A64, 112.

(12) Dolomanov, O. V.; Bourhis, L. J.; Gildea, R. J.; Howard, J. A. K.; Puschmann, H. OLEX2: a complete structure solution, refinement and analysis program. J. Appl. Cryst. 2009, 42, 339. 
(13) Farrugia, L. J. WinGX and ORTEP for Windows: an update. J. Appl. Cryst. 2012, 45, 849.

(14) Macrae, C. F.; Bruno, I. J.; Chisholm, J. A.; Edgington, P. R.; McCabe, P.; Pidcock, E.; Rodriguez-Monge, L.; Taylor, R.; van de Streek, J.; Wood, P. A. Mercury CSD 2.0 - new features for the visualization and investigation of crystal structures. J. Appl. Cryst. 2008, 41, 466.

(15) Lohrey, T. D.; Rao, G.; Britt, R. D.; Bergman, R. G.; Arnold, J. Manuscript in preparation.

(16) Gaussian 09, Revision D.01. Frisch, M. J.; Trucks, G. W.; Schlegel, H. B.; Scuseria, G. E.; Robb, M. A.; Cheeseman, J. R.; Scalmani, G.; Barone, V.; Mennucci, B.; Petersson, G. A.; Nakatsuji, H.; Caricato, M.; Li, X.; Hratchian, H. P.; Izmaylov, A. F.; Bloino, J.; Zheng, G.; Sonnenberg, J. L.; Hada, M.; Ehara, M.; Toyota, K.; Fukuda, R.; Hasegawa, J.; Ishida, M.; Nakajima, T.; Honda, Y.; Kitao, O.; Nakai, H.; Vreven, T.; Montgomery, J. A., Jr.; Peralta, J. E.; Ogliaro, F.; Bearpark, M.; Heyd, J. J.; Brothers, E.; Kudin, K. N.; Staroverov, V. N.; Kobayashi, R.; Normand, J.; Raghavachari, K.; Rendell, A.; Burant, J. C.; Iyengar, S. S.; Tomasi, J.; Cossi, M.; Rega, N.; Millam, J. M.; Klene, M.; Knox, J. E.; Cross, J. B.; Bakken, V.; Adamo, C.; Jaramillo, J.; Gomperts, R.; Stratmann, R. E.; Yazyev, O.; Austin, A. J.; Cammi, R.; Pomelli, C.; Ochterski, J. W.; Martin, R. L.; Morokuma, K.; Zakrzewski, V. G.; Voth, G. A.; Salvador, P.; Dannenberg, J. J.; Dapprich, S.; Daniels, A. D.; Farkas, Ö.; Foresman, J. B.; Ortiz, J. V.; Cioslowski, J.; Fox, D. J. Gaussian, Inc., Wallingford CT, 2009.

(17) Becke, A. D. Density-functional thermochemistry. III. The role of exact exchange. J. Chem. Phys. 1993, 98, 5648.

(18) Perdew, J. P.; Wang, Y. Accurate and simple analytic representation of the electron-gas correlation energy. Phys. Rev. B 1992, 45, 13244.

(19) Grimme, S.; Ehrlich, S; Goerigk, L. J. Comp. Chem. 2011, 32, 1456.

(20) Dolg, M.; Wedig, U.; Stoll, H.; Preuß, H. Energy-adjusted ab initio pseudopotentials for the first row transition elements. J. Chem. Phys. 1987, 86, 866.

(21) Dolg, M. Effective Core Potentials. In Modern Methods and Algorithm of Quantum Chemistry; Grotendorst, J., Ed.; John von Neuman Institute for Computing: Jülich, Germany, 2000; Vol. 1, p 479.

(22) Andrae, D.; Häeußermann, U.; Dolg, M.; Stoll, H.; Preuß, H. Energy-adjusted ab initio pseudopotentials for the second and third row transition elements. Theor. Chim. Acta 1990, 77, 123. 
(23) Ehlers, A. W.; Böhme, M.; Dapprich, S.; Gobbi, A.; Höllwarth, A.; Jonas, V.; Köhler, K. F.; Stegmann, R.; Veldkamp, A.; Frenking, G. A set of f-polarization functions for pseudo-potential basis sets of the transition metals $\mathrm{Sc}-\mathrm{Cu}, \mathrm{Y}-\mathrm{Ag}$ and La-Au. Chem. Phys. Lett. 1993, $208,111$.

(24) Hariharan, P. C.; Pople, J. A. The Influence of Polarization Functions on Molecular Orbital Hydrogenation Energies. Theor. Chim. Acta 1973, 28, 213.

(25) Reed, A. E.; Curtiss, L. A.; Weinhold, F. Intermolecular Interactions from a Natural Bond Orbital, Donor-Acceptor Viewpoint. Chem. Rev. 1988, 88, 899.

(26) Weinhold, F. Natural bond orbital methods. In Encyclopedia of Computational Chemistry; Schleyer, P. v. R., Allinger, N. L., Clark, T., Gasteiger, J., Kollman, P. A., Schaefer, H. F., III, Schreiner, P. R., Eds.; John Wiley \& Sons: Chichester, UK, 1998; Vol. 3 p 1792.

(27) www.chemcraftprog.com 